\title{
On the geometry and stability of phosphatidylcholine-based bioclays
}

\author{
P. GRANČIČ ${ }^{1}$ AND DANIEL TUNEGA ${ }^{1,2}$
}

${ }^{1}$ University of Natural Resources and Life Sciences, PeterJordan-Strasse 82b, A-1190 Wien, Austria

(peter.grancic@boku.ac.at, daniel.tunega@boku.ac.at)

${ }^{2}$ School of Pharmaceutical Science and Technology, Tianjin University, Tianjin, 300072, P. R. China

A class of new perspective materials (here referred to as bioclays) can be prepared by combining clay minerals with charged bioorganic moieties. This work presents a comprehensive computational study of phosphatidylcholinebioclay composite models by employing a series of classical molecular dynamics simulations. Our detailed analysis of the structure and energies of the resulting bioclays reveals that the phosphatidylcholine molecules bind to the clay surfaces through their zwitterionic heads, forming layers with the aliphatic tails stretched preferably parallel to the clay surface. The tails exhibit varying degrees of flexibility and disorder depending on their surface loading and distance from the clay surface. The observed detachment of naturally occurring sodium cations in the case of montmorillonite surfaces caused by the presence of the phosphatidylcholine zwitterionic heads suggests that cation-exchange is very likely the driving mechanism for the phosphatidylcholine adsorption on montmorillonite. Once the first layer of phosphatidylcholine forms, more molecules can still be attached and stabilised by their mutual intermolecular interactions.

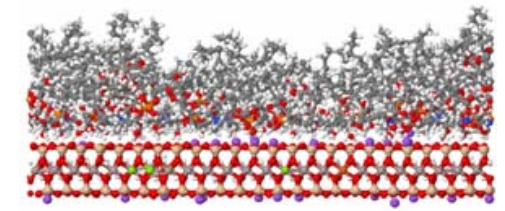

Figure 1: phosphatidylcholine-montmorillonite bioclay (snapshot from molecular dynamics).

Acknowledgements: PG and DT are grateful for the financial support by the Austrian Grand Agency (FWF), project No. I3263-N34. 